\title{
EQUICONNECTED SPACES AND BAIRE CLASSIFICATION OF SEPARATELY CONTINUOUS FUNCTIONS AND THEIR ANALOGS
}

\author{
OLENA KARLOVA, VOLODYMYR MASLYUCHENKO, AND VOLODYMYR MYKHAYLYUK
}

\begin{abstract}
We investigate the Baire classification of mappings $f: X \times Y \rightarrow Z$, where $X$ belongs to a wide class of spaces, which includes all metrizable spaces, $Y$ is a topological space, $Z$ is an equiconnected space, which are continuous in the first variable and for a dense set in $X$ these mappings are functions of a Baire class $\alpha$ in the second variable.
\end{abstract}

\section{INTRODUCTION}

We will denote by $P(X, Y)$ the collection of all mappings $f: X \rightarrow Y$ with a property $P$. For a mapping $f: X \times Y \rightarrow Z$ and a point $(x, y) \in X \times Y$ write $f^{x}(y)=f_{y}(x)=f(x, y)$.

Let $P$ and $Q$ be some properties of mappings. Set

$$
\begin{gathered}
X_{Q}(f)=\left\{x \in X: f^{x} \in Q(Y, Z)\right\} ; \quad Y_{P}(f)=\left\{y \in Y: f_{y} \in P(X, Z)\right\} ; \\
P Q(X \times Y, Z)=\left\{f \in Z^{X \times Y}: X_{Q}(f)=X, Y_{P}(f)=Y\right\} ; \\
P \bar{Q}(X \times Y, Z)=\left\{f \in Z^{X \times Y}: \overline{X_{Q}(f)}=X, Y_{P}(f)=Y\right\} .
\end{gathered}
$$

Let $C(X, Y)$ denote the collection of all continuous mappings between $X$ and $Y$. A mapping $f: X \times Y \rightarrow Z$ is separately continuous if $f \in C C(X \times Y, Z)$. We call a mapping $f: X \times Y \rightarrow Z$ vertically nearly separately continuous if $f \in C \bar{C}(X \times Y, Z)$.

A mapping $f: X \rightarrow Y$ is said to be a mapping of the first Baire class or a Baire-one mapping if there exists a sequence of continuous mappings $f_{n}: X \rightarrow Y$ such that $f_{n}(x) \rightarrow f(x)$ for every $x \in X$. The class of all Baire-one mappings $f: X \rightarrow Y$ will be denoted by $B_{1}(X, Y)$. Let $\alpha>0$ be an at most countable ordinal and assume that classes $B_{\xi}(X, Y)$ are already defined for all $\xi<\alpha$. A mapping $f: X \rightarrow Y$ belongs to the $\alpha$-th Baire class, $f \in B_{\alpha}(X, Y)$, if there exists a sequence of mappings $f_{n} \in \bigcup_{\xi<\alpha} B_{\xi}(X, Y)$ such that $f_{n}(x) \rightarrow f(x)$ for every $x \in X$. Notice that for an arbitrary sequence $\left(\alpha_{n}\right)_{n=1}^{\infty}$ of ordinals $\alpha_{n}<\alpha$ such that $\lim _{n \rightarrow \infty}\left(\alpha_{n}+1\right)=\alpha$, we can choose a sequence $\left(f_{n}\right)_{n=1}^{\infty}$ in such a way that $f_{n} \in B_{\alpha_{n}}(X, Y)$ for every $n \in \mathbb{N}$. Define $B_{0}(X, Y)=C(X, Y)$.

In $1898 \mathrm{H}$. Lebesgue [8] proved that every real-valued separately continuous functions of two real variables is of the first Baire class. In honour of his theorem, we call a collection $(X, Y, Z)$ of topological spaces a Lebesgue $\alpha$-triple, if $C B_{\alpha}(X \times Y, Z) \subseteq B_{\alpha+1}(X \times Y, Z)$, where $0 \leq \alpha<\omega_{1}$.

Lebesgue's theorem was generalized by many mathematicians (see [5, 9, 11, 14, 13, 1, 2, 6, 7, 10] and the references given there). The classical work here is the paper of W. Rudin [11, who proved the following theorem.

Theorem 1.1. Let $X$ be a metrizable space, $Y$ a topological space and $Z$ a locally convex space. Then $C \bar{C}(X \times$ $Y, Z) \subseteq B_{1}(X \times Y, Z)$.

A collection $(X, Y, Z)$ of topological spaces is called a Rudin $\alpha$-triple, if $C \bar{B}_{\alpha}(X \times Y, Z) \subseteq B_{\alpha+1}(X \times Y, Z)$, where $0 \leq \alpha<\omega_{1}$.

The following question is still unanswered.

Problem 1.2. Do there exist a metrizable space $X$, a topological space $Y$ and a topological vector space (or, more general, an equiconnected space) $Z$ such that $(X, Y, Z)$ is not a Rudin 0 -triple?

The following result was proved by V. Maslyuchenko and A. Kalancha [6] .

Theorem 1.3. Let $X$ be a metrizable space with finite Čech-Lebesgue dimension, $Y$ a topological space, $Z$ a topological vector space and $0 \leq \alpha<\omega_{1}$. Then $(X, Y, Z)$ is a Rudin $\alpha$-triple.

In [13] O. Sobchuk introduced the class of PP-spaces (see definition in Section 4), which includes the class of all metrizable spaces, and obtained the following fact (see [12]).

Theorem 1.4. A collection $(X, Y, Z)$ is a Lebesgue $\alpha$-triple if $X$ is a PP-space, $Y$ is a topological space and $Z$ is a locally convex space. 
The following two results were established by T. Banakh [1, who introduced metrically quarter-stratifiable spaces and studied their applications to Baire classification of separately continuous functions with values in equiconnected spaces (see definitions in Section 2 and Section 44).

Theorem 1.5. Let $X$ be a metrically quarter-stratifiable space, $Y$ a topological space and $Z$ an equiconnected space. If, moreover, $X$ is paracompact and strongly countable-dimensional or $Z$ is locally convex, then $(X, Y, Z)$ is a Lebesgue 0-triple.

Theorem 1.6. Let $1 \leq \alpha<\omega_{1}, X$ a metrically quarter-stratifiable space, $Y$ a topological space and $Z$ a contractible space. Then $(X, Y, Z)$ is a Lebesgue $\alpha$-triple.

Obviously, every Rudin $\alpha$-triple is a Lebesgue $\alpha$-triple. In [1, Example 5.7] was given an example which shows that Theorems 1.4 and 1.5 cannot be generalized to the case when $(X, Y, Z)$ is a Rudin 0-triple. Therefore, it is naturally to ask if we can replace Lebesgue triple with a Rudin triple in Theorems 1.4 and 1.6, and under which assumptions on $X$ analogs of Theorems 1.3, 1.4, 1.5 and 1.6 take place? To answer these questions, we first introduced notions of a convex combination and an $\lambda$-sum of elements of an equiconnected space (see Sections 2 and 3) and describe some of their properties. In Section 4 we introduce the class of strong PP-spaces and find out that $(X, Y, Z)$ is a Rudin $\alpha$-triple for $\alpha \geq 0$ if $X$ is a strong PP-space, $Y$ is a topological space and $Z$ is a locally convex equiconnected space. Further, in Section 5 we prove that $(X, Y, Z)$ is a Rudin $\alpha$-triple for $\alpha \geq 0$ if $X$ is a paracompact Hausdorff strongly countable-dimensional strong PP-space, $Y$ is a topological space and $Z$ is an equiconnected space. This gives the generalization of Theorem 1.3 for equiconnected space $Z$ and for Rudin triples. Functions of the classes $C \bar{B}_{\alpha}$ with $\alpha>0$, are investigated in Section 6 . There we show that if $X$ is a strong PP-space, $Y$ is a topological space and $Z$ is a conractible space, then $(X, Y, Z)$ is a Rudin $\alpha$-triple. Finally, in Section 7 we give several examples of PP-spaces which are not strong PP-spaces, consequently, these examples show that Theorem 1.6 cannot be generalized to Rudin triples.

\section{Convex COMbinations in AN EQuiconneCted SPACE AND THEir Properties}

Definition 2.1. An equiconnected space is a pair $(X, \lambda)$, consisting of a topological space $X$ and a continuous function $\lambda: X \times X \times[0,1] \rightarrow X$ such that

(i) $\lambda(x, y, 0)=x$;

(ii) $\lambda(x, y, 1)=y$;

(iii) $\lambda(x, x, t)=x$

for all $x, y \in X$ and $t \in[0,1]$.

The simplest example of an equiconnected space is a convex subset of a topological vector space, where $\lambda$ can be defined as $\lambda(x, y, t)=(1-t) x+t y$.

For every $n \in \mathbb{N}$ denote

$$
S_{n}=\left\{\alpha=\left(\alpha_{1}, \ldots, \alpha_{n}\right) \in \mathbb{R}^{n}: \alpha_{1}, \ldots, \alpha_{n} \geq 0, \alpha_{1}+\cdots+\alpha_{n}=1\right\} .
$$

Let $(X, \lambda)$ be an equiconnected space. By induction in $n \in \mathbb{N}$ we define a sequence $\left(\lambda_{n}\right)_{n=1}^{\infty}$ of mappings $\lambda_{n}: X^{n} \times S_{n} \rightarrow X$.

For $n=1$ set $\lambda_{1}(x, 1)=x$ for every $x \in X$. Now for $n \in \mathbb{N}, x_{1}, \ldots x_{n+1} \in X$ and $\left(\alpha_{1}, \ldots, \alpha_{n+1}\right) \in S_{n+1}$ let

$$
\begin{gathered}
\lambda_{n+1}\left(x_{1}, \ldots x_{n+1}, \alpha_{1}, \ldots, \alpha_{n+1}\right)= \\
=\lambda_{n}\left(\lambda\left(x_{1}, x_{2}, \frac{\alpha_{2}}{\alpha_{1}+\alpha_{2}}\right), x_{3}, \ldots x_{n+1}, \alpha_{1}+\alpha_{2}, \alpha_{3} \ldots, \alpha_{n+1}\right), \text { if } \alpha_{1}+\alpha_{2}>0 ; \\
\lambda_{n+1}\left(x_{1}, \ldots x_{n+1}, \alpha_{1}, \ldots, \alpha_{n+1}\right)= \\
=\lambda_{n}\left(x_{2}, x_{3}, \ldots x_{n+1}, \alpha_{2}, \alpha_{3} \ldots, \alpha_{n+1}\right), \text { if } \alpha_{1}+\alpha_{2}=0 .
\end{gathered}
$$

Definition 2.2. For $n \in \mathbb{N},\left(\alpha_{1}, \ldots, \alpha_{n}\right) \in S_{n}$ and any elements $x_{1}, \ldots x_{n}$ of an equiconnected space $(X, \lambda)$ the element $\lambda_{n}\left(x_{1}, \ldots x_{n}, \alpha_{1}, \ldots, \alpha_{n}\right)$ is called a convex combination of the elements $x_{1}, \ldots x_{n}$ with the coefficients $\alpha_{1}, \ldots, \alpha_{n}$.

Proposition 2.3. Let $(X, \lambda)$ be an equiconnected space. Then $\left(\lambda_{n}\right)_{n=1}^{\infty}$ has the following properties:

(i) if $n \geq 2,1 \leq k \leq n$ and $\alpha_{k}=0$, then

$$
\begin{gathered}
\lambda_{n}\left(x_{1}, \ldots x_{n}, \alpha_{1}, \ldots, \alpha_{n}\right)= \\
=\lambda_{n-1}\left(x_{1}, \ldots x_{k-1}, x_{k+1} \ldots x_{n}, \alpha_{1}, \ldots \alpha_{k-1}, \alpha_{k+1} \ldots \alpha_{n}\right)
\end{gathered}
$$

for any $x_{1}, \ldots x_{n} \in X$ and $\left(\alpha_{1}, \ldots, \alpha_{n}\right) \in S_{n}$

(ii) $\lambda_{n}\left(x, \ldots x, \alpha_{1}, \ldots, \alpha_{n}\right)=x$ for any $x \in X$ and $\left(\alpha_{1}, \ldots, \alpha_{n}\right) \in S_{n}$;

(iii) every $\lambda_{n}$ is continuous. 
Proof. By induction on $n$ properties (i) and (ii) easily follow from the definition of functions $\lambda_{n}$.

(iii). Clearly, $\lambda_{1}$ is continuous. Assume that functions $\lambda_{1}, \ldots, \lambda_{n}$ are continuous and show that $\lambda_{n+1}$ is continuous.

Fix points $x_{1}, \ldots, x_{n+1} \in X$ and a vector $\left(\alpha_{1}, \ldots, \alpha_{n+1}\right) \in S_{n+1}$, and prove that $\lambda_{n+1}$ is continuous at $\left(x_{1}, \ldots, x_{n+1}, \alpha_{1}, \ldots, \alpha_{n+1}\right)$.

Let

$$
\begin{aligned}
G=\left\{\left(y_{1}, \ldots y_{n+1}, \beta_{1}, \ldots \beta_{n+1}\right)\right. & : y_{1}, \ldots y_{n+1} \in X \\
& \left.\left(\beta_{1}, \ldots \beta_{n+1}\right) \in S_{n+1}, \beta_{1}+\beta_{2}>0\right\} .
\end{aligned}
$$

Since $G$ is open and functions $\lambda$ and $\lambda_{n}$ are continuous, $\lambda_{n+1}$ is continuous at every point of $G$, in particular, $\lambda_{n+1}$ is continuous at $\left(x_{1}, \ldots, x_{n+1}, \alpha_{1}, \ldots, \alpha_{n+1}\right)$ if $\alpha_{1}+\alpha_{2}>0$.

Now let $\alpha_{1}=\alpha_{2}=0, z=\lambda_{n+1}\left(x_{1}, \ldots, x_{n+1}, \alpha_{1}, \ldots, \alpha_{n+1}\right)$ and let $W$ be an open neighborhood of $z$ in $X$. Consider the set $A=\left\{\lambda\left(x_{1}, x_{2}, \alpha\right): 0 \leq \alpha \leq 1\right\}$. Observe that $A$ is compact and $\lambda_{n}\left(a, x_{3}, \ldots, x_{n+1}, 0, \alpha_{3}, \ldots, \alpha_{n+1}\right)=z$ for every $a \in A$ by $(i)$. Since $\lambda_{n}$ is continuous, there exist an open set $U$ in $X$ with $A \subseteq U$, open neighborhoods $U_{3}, \ldots, U_{n+1}$ in $X$ of points $x_{3}, \ldots, x_{n+1}$, respectively, and open neighborhoods $V, V_{3}, \ldots, V_{n+1}$ of points $0, \alpha_{3}, \ldots, \alpha_{n+1}$ in $[0,1]$ such that $\lambda_{n}\left(y, z_{3}, \ldots z_{n+1}, \gamma, \beta_{3}, \ldots, \beta_{n+1}\right) \in W$ for any $y \in U, z_{3} \in U_{3}, \ldots, z_{n+1} \in U_{n+1}$ and $\gamma \in V, \beta_{3} \in V_{3}, \ldots, \beta_{n+1} \in V_{n+1}$. Now, taking into account that $A \subseteq U$ and that $\lambda_{2}$ is continuous, we choose neighborhoods $U_{1}$ and $U_{2}$ of $x_{1}$ and $x_{2}$ such that $\lambda\left(z_{1}, z_{2}, \beta\right) \in U$ for any $z_{1} \in U_{1}, z_{2} \in U_{2}$ and $\beta \in[0,1]$. Moreover, choose neighborhoods $V_{1}$ and $V_{2}$ of 0 in $[0,1]$ such that $V_{1}+V_{2} \subseteq V$. Show that

$$
\lambda_{n+1}\left(z_{1}, \ldots z_{n+1}, \beta_{1}, \ldots, \beta_{n+1}\right) \in W
$$

for any $z_{1} \in U_{1}, \ldots z_{n+1} \in U_{n+1}$ and $\beta_{1} \in V_{1}, \ldots, \beta_{n+1} \in V_{n+1}$.

If $\beta_{1}=\beta_{2}=0$, then, taking into account that $z_{2} \in U$ and $0 \in V$, we conclude that

$$
\lambda_{n+1}\left(z_{1}, \ldots z_{n+1}, \beta_{1}, \ldots, \beta_{n+1}\right)=\lambda_{n}\left(z_{2}, z_{3} \ldots z_{n+1}, 0, \beta_{3}, \ldots, \beta_{n+1}\right) \in W .
$$

If $\beta_{1}+\beta_{2}>0$, then

$$
\lambda_{n+1}\left(z_{1}, \ldots z_{n+1}, \beta_{1}, \ldots, \beta_{n+1}\right)=\lambda_{n}\left(y, z_{3} \ldots z_{n+1}, \gamma, \beta_{3}, \ldots, \beta_{n+1}\right) \in W
$$

where $y=\lambda\left(z_{1}, z_{2}, \frac{\beta_{2}}{\beta_{1}+\beta_{2}}\right) \in U$ and $\gamma=\beta_{1}+\beta_{2} \in V_{1}+V_{2} \subseteq V$.

Let $(X, \lambda)$ be an equiconnected space and $A \subseteq X$ a nonempty set. Put $\lambda^{0}(A)=A, \lambda^{n}(A)=\lambda\left(\lambda^{n-1}(A) \times A \times\right.$ $[0,1])$ for $n \in \mathbb{N}$ and $\lambda^{\infty}(A)=\bigcup_{n=1}^{\infty} \lambda^{n}(A)$. According to [1] we call an equiconnected space $(X, \lambda)$ locally convex if for every $x \in X$ and a neighborhood $U$ of $x$ in $X$ there exists a neighborhood $V$ of $x$ such that $\lambda^{\infty}(V) \subseteq U$.

The following fact directly follows from the definitions.

Proposition 2.4. Let $(X, \lambda)$ be an equiconnected space, $A \subseteq X$ and $n \in \mathbb{N}$. Then

$$
\lambda^{n}(A)=\left\{\lambda_{n}\left(x_{1}, \ldots x_{n}, \alpha_{1}, \ldots, \alpha_{n}\right):\left(x_{1}, \ldots x_{n}\right) \in A^{n},\left(\alpha_{1}, \ldots, \alpha_{n}\right) \in S_{n}\right\} .
$$

\section{3. $\lambda$-SUMS AND BAIRE MEASURABle FUNCTIONS}

Definition 3.1. Let $(I, \leq)$ be a well ordered set, $\left(x_{i}\right)_{i \in I}$ a family of elements $x_{i}$ of an equiconnected space $(X, \lambda)$, and $\left(\alpha_{i}\right)_{i \in I}$ a collection of scalars $\alpha_{i} \geq 0$ such that:

(1) $\left\{i \in I: \alpha_{i} \neq 0\right\}=\left\{i_{k}: 1 \leq k \leq n\right\}$;

(2) $i_{1}<i_{2}<\cdots<i_{n}$;

(3) $\alpha_{i_{1}}+\alpha_{i_{2}}+\cdots+\alpha_{i_{n}}=1$.

Then the element $\lambda_{n}\left(x_{i_{1}}, \ldots, x_{i_{n}}, \alpha_{i_{1}}, \ldots, \alpha_{i_{n}}\right)$ is called a $\lambda$-sum of elements $\left(x_{i}\right)_{i \in I}$ with coefficients $\left(\alpha_{i}\right)_{i \in I}$ and is denoted by $\sum_{i \in I}^{\lambda} \alpha_{i} x_{i}$.

Let us observe that $\sum_{i \in I}^{\lambda} \alpha_{i} x_{i}=\sum_{i \in I} \alpha_{i} x_{i}$ for an arbitrary topological vector space $X$, where $\lambda(x, y, t)=$ $(1-t) x+t y$.

Theorem 3.2. Let $X$ and $Y$ be topological spaces, $(Z, \lambda)$ an equiconnected space, $(I, \leq)$ a well ordered set, let $0 \leq \alpha<\omega_{1},\left(f_{i}\right)_{i \in I}$ a family of mappings $f_{i}: Y \rightarrow Z$ of Baire class $\alpha$ and $\left(\varphi_{i}\right)_{i \in I}$ a locally finite partition of unity on $X$. Then a mapping $f: X \times Y \rightarrow Z, f(x, y)=\sum_{i \in I}^{\lambda} \varphi_{i}(x) f_{i}(y)$, is of the Baire class $\alpha$; in particular, if all mappings $f_{i}$ are continuous, then so is $f$. 
Proof. We will argue by induction in $\alpha$. We first consider the case $\alpha=0$, i.e. the case when all mappings $f_{i}$ are continuous. Fix a point $x_{0} \in X$ and show that $f$ is continuous at every point of $\left\{x_{0}\right\} \times Y$.

Using locally finiteness of partition of unity $\left(\varphi_{i}\right)_{i \in I}$, we choose an open neighborhood $U$ of $x_{0}$ in $X$ such that $I_{0}=\left\{i \in I: \operatorname{supp} \varphi_{n} \cap U \neq \varnothing\right\}$ is finite. Let $I_{0}=\left\{i_{k}: 1 \leq k \leq n\right\}$ and $i_{1}<i_{2}<\cdots<i_{n}$. The definition of $\lambda$-sum and Proposition $2.3(i)$ imply

$$
f(x, y)=\lambda_{n}\left(f_{i_{1}}(y), \ldots, f_{i_{n}}(y), \varphi_{i_{1}}(x), \ldots, \varphi_{i_{n}}(x)\right)
$$

for any $x \in U$ and $y \in Y$. Since mappings $\lambda_{n}, f_{i}$ and $\varphi_{i}$ are continuous, $f$ is continuous on $U \times Y$; in particular, $f$ is continuous at every point of $\left\{x_{0}\right\} \times Y$. Hence, the theorem is true for $\alpha=0$.

Now suppose that the theorem is true for all ordinals $\alpha<\beta$ with some fixed ordinal $0<\beta<\omega_{1}$. Let $\left(f_{i}\right)_{i \in I}$ be a family of mappings $f_{i} \in B_{\beta}(Y, Z)$. Choose an increasing sequence $\left(\alpha_{n}\right)_{n=1}^{\infty}$ of ordinals $\alpha_{n}<\beta$ such that $\lim _{n \rightarrow \infty}\left(\alpha_{n}+1\right)=\beta$ and a sequence of families $\left(f_{i, n}\right)_{i \in I}$ of mappings $f_{i, n} \in B_{\alpha_{n}}(Y, Z)$ such that $f_{i, n}(y) \rightarrow f_{i}(y)$ for all $y \in Y$ and $i \in I$. By the assumption, the mapping $g_{n}: X \times Y \rightarrow Z, g_{n}(x, y)=\sum_{i \in I}^{\lambda} \varphi_{i}(x) f_{i, n}(y)$, is of the class $\alpha_{n}$ for every $n \in \mathbb{N}$. Moreover, the continuity of $\lambda_{n}$ implies $g_{n}(x, y) \rightarrow f(x, y)$ for all $x \in X$ and $y \in Y$.

\section{Strong PP-Spaces And MAPpings into LOCAlly CONVEX SPACES}

Definition 4.1. A topological space $(X, \mathcal{T})$ is called quarter-stratifiable if there exists a function $g: \mathbb{N} \times X \rightarrow \mathcal{T}$ (called quarter-stratifying function) such that

(1) $X=\bigcup_{x \in X} g(n, x)$ for every $n \in \mathbb{N}$;

(2) $\left(x \in g\left(n, x_{n}\right), n \in \mathbb{N}\right) \Rightarrow\left(x_{n} \rightarrow x\right)$.

A topology $\mathcal{T}^{\prime}$ on a topological space $(X, \mathcal{T})$ is called quarter-stratifying if there exists a quarter-stratifying function $g: \mathbb{N} \times X \rightarrow \mathcal{T}$ for $X$ such that $g(\mathbb{N} \times X) \subseteq \mathcal{T}^{\prime}$.

A topological space $X$ is said to be metrically quarter-stratifiable if it admits a weaker metrizable quarterstratifying topology.

The topology on a topological space $X$ we denote by $\mathcal{T}(X)$.

Definition 4.2. A topological space $X$ is called (strong) PP-space if (for any dense set $D \subseteq X$ ) there exist a sequence $\left(\mathcal{U}_{n}\right)_{n=1}^{\infty}$ of $\left(\left(\varphi_{i, n}: i \in I_{n}\right)\right)_{n=1}^{\infty}$ of locally finite partitions of unity on $X$ and a sequence $\left(\left(x_{i, n}: i \in\right.\right.$ $\left.\left.I_{n}\right)\right)_{n=1}^{\infty}$ of families of points of $X$ (of $D$ ) such that

$$
(\forall x \in X)(\forall U \ni x, U \in \mathcal{T}(X))\left(\exists n_{0} \in \mathbb{N}\right)\left(\forall n \geq n_{0}\right)\left(\forall i \in I_{n}\right)
$$

$$
\left(x \in \operatorname{supp} \varphi_{i, n} \Longrightarrow x_{i, n} \in U\right)
$$

Obviously, every strong PP-space is a PP-space.

\section{Remark 4.3.}

(1) Every metrically quarter-stratifiable space is a PP-space according to [10, Remark 3.1].

(2) Every Hausdorff PP-space is metrically quarter stratifiable by [10, Proposition 3.2].

It is easy to see that any metrizable space, or, more general, every space equipped with a topology generated by a pseudo-metric, is strong PP-space. Therefore, a topological space $X$ equipped with the topology generated by a pseudo-metric which is not a metric on $X$, is a PP-space, but is not metrically quarter-stratifiable.

The following proposition gives an example of a non-metrizable strong PP-space.

Proposition 4.4. The Sorgenfrey line $\mathbb{L}$ is a strong PP-space.

Proof. Let $D \subseteq \mathbb{L}$ be a dense set. For any $n \in \mathbb{N}$ and $i \in \mathbb{Z}$ denote by $\varphi_{i, n}$ the characteristic function of $\left[\frac{i-1}{n}, \frac{i}{n}\right)$ and choose a point $x_{i, n} \in\left[\frac{i}{n}, \frac{i+1}{n}\right) \cap D$. Then the sequences $\left(\left(\varphi_{i, n}: i \in I_{n}\right)\right)_{n=1}^{\infty}$ and $\left(\left(x_{i, n}: i \in I_{n}\right)\right)_{n=1}^{\infty}$ satisfy (4.1).

Examples of PP-spaces which are not strong PP-spaces can be found in Section 7 (see Examples 7.1 and 7.3).

Theorem 4.5. Let $X$ be a strong PP-space, $Y$ a topological space, $(Z, \lambda)$ a locally convex equiconnected space and $0 \leq \alpha<\omega_{1}$. Then $(X, Y, Z)$ is a Rudin $\alpha$-triple. 
Proof. Let $f \in C \bar{B}_{\alpha}(X \times Y, Z)$. Since $X_{B_{\alpha}}(f)$ is dense in $X$, we can choose a sequence $\left(\left(\varphi_{i, n}: i \in I_{n}\right)\right)_{n=1}^{\infty}$ of locally finite partitions of unity on $X$ and a sequence $\left(\left(x_{i, n}: i \in I_{n}\right)\right)_{n=1}^{\infty}$ of families of points of $X_{B_{\alpha}}(f)$ which satisfy (4.1).

For every $n \in \mathbb{N}$ we well order the set $I_{n}$ and for all $(x, y) \in X \times Y$ we set

$$
f_{n}(x, y)=\sum_{i \in I_{n}}^{\lambda} \varphi_{i, n}(x) f\left(x_{i, n}, y\right)
$$

Then $f^{x_{i, n}} \in B_{\alpha}(Y, Z)$ for every pair $(i, n) \in I_{n} \times \mathbb{N}$. Therefore, applying Theorem 3.2, we have that $f_{n} \in$ $B_{\alpha}(X \times Y, Z)$ for every $n \in \mathbb{N}$.

We will show that $f_{n}(x, y) \rightarrow f(x, y)$ on $X \times Y$. Fix $\left(x_{0}, y_{0}\right) \in X \times Y$ and consider an arbitrary neighborhood $W$ of $z_{0}=f\left(x_{0}, y_{0}\right)$ in $Z$. Since $Z$ is locally convex, there is a neighborhood $W_{1}$ of $z_{0}$ such that $\lambda^{\infty}\left(W_{1}\right) \subseteq W$. The continuity of $f$ in the first variable at $\left(x_{0}, y_{0}\right)$ implies the existence of a neighborhood $U$ of $x_{0}$ in $X$ such that $f\left(x, y_{0}\right) \in W_{1}$ for every $x \in U$. Now, using (4.1), we choose a number $n_{0}$ such that $x_{i, n} \in U$ for all $n \geq n_{0}$ and $i \in I_{n}$ with $x_{0} \in \operatorname{supp} \varphi_{i, n}$.

Now we prove that $f_{n}\left(x_{0}, y_{0}\right) \in W$ for all $n \geq n_{0}$. Fix $n \geq n_{0}$ and set

$$
I=\left\{i \in I_{n}: x_{0} \in \operatorname{supp} \varphi_{i, n}\right\} \text {. }
$$

Let $I=\left\{i_{1}, i_{2}, \ldots i_{k}\right\}$, where $i_{1}<i_{2}<\cdots<i_{k}$. Denote $\alpha_{1}=\varphi_{i_{1}, n}\left(x_{0}\right), \ldots, \alpha_{k}=\varphi_{i_{k}, n}\left(x_{0}\right), z_{1}=$ $f\left(x_{i_{1}, n}, y_{0}\right), \ldots, z_{k}=f\left(x_{i_{k}, n}, y_{0}\right)$. Then $z_{1}, \ldots z_{k} \in W_{1}$ and

$$
f_{n}\left(x_{0}, y_{0}\right)=\lambda_{k}\left(z_{1}, \ldots, z_{k}, \alpha_{1}, \ldots \alpha_{k}\right) \in \lambda^{k}\left(W_{1}\right) \subseteq W .
$$

Corollary 4.6. Let $X$ be a metrizable space, $Y$ a topological space, $(Z, \lambda)$ a locally convex equiconnected space. Then $(X, Y, Z)$ is a Rudin $\alpha$-triple for every $0 \leq \alpha<\omega_{1}$.

\section{Mappings into EQUiCONNECTED SPACES}

Definition 5.1. A sequence $\left(\mathcal{A}_{n}\right)_{n=1}^{\infty}$ of families $\mathcal{A}_{n}=\left(A_{i, n}: i \in I_{n}\right)$ of subsets $A_{i, n}$ of a topological space $X$ is called uniformly pointwise finite in $X$ if for every $x \in X$ there exists $k_{x} \in \mathbb{N}$ such that for every $n \in \mathbb{N}$ the set $\left\{i \in I_{n}: x \in A_{i, n}\right\}$ contains at most $k_{x}$ elements.

Theorem 5.2. Let $X$ and $Y$ be topological spaces, $(Z, \lambda)$ an equiconnected space, $f: X \times Y \rightarrow Z$ a continuous mapping in the first variable, $0 \leq \alpha<\omega_{1}$; let $\left(\left(\varphi_{i, n}: i \in I_{n}\right)\right)_{n=1}^{\infty}$ be a sequence of locally finite partitions of unity on $X$ and $\left(\left(x_{i, n}: i \in I_{n}\right)\right)_{n=1}^{\infty}$ a sequence of families of points $x_{i, n} \in X_{B_{\alpha}}(f)$ such that 4.1) holds, and let the sequence $\left(\mathcal{A}_{n}\right)_{n=1}^{\infty}$ of families $\mathcal{A}_{n}=\left(\operatorname{supp} \varphi_{i, n}: i \in I_{n}\right)$ be uniformly pointwise finite in $X$. Then $f \in B_{\alpha+1}(X \times Y, Z)$.

Proof. For every $n \in \mathbb{N}$ we well order the set $I_{n}$ and for all $(x, y) \in X \times Y$ let

$$
f_{n}(x, y)=\sum_{i \in I_{n}}^{\lambda} \varphi_{i, n}(x) f\left(x_{i, n}, y\right) .
$$

Analogously as in the proof of Theorem 4.5 we have $f_{n} \in B_{\alpha}(X \times Y, Z)$ for every $n \in \mathbb{N}$.

It remains to prove that $f_{n}(x, y) \rightarrow f(x, y)$ on $X \times Y$. Fix $\left(x_{0}, y_{0}\right) \in X \times Y$ and consider a neighborhood $W$ of $z_{0}=f\left(x_{0}, y_{0}\right)$ in $Z$. Denote by $m$ the number $k_{x_{0}}$ from Definition 5.1. Since $\lambda_{m}$ is continuous and $\lambda_{m}\left(z_{0}, \ldots, z_{0}, \alpha_{1}, \ldots, \alpha_{m}\right)=z_{0}$ for all $\left(\alpha_{1}, \ldots, \alpha_{m}\right) \in S_{m}$ and $S_{m}$ is compact, there exists a neighborhood $W_{1}$ of $z_{0}$ in $Z$ such that $\lambda_{m}\left(z_{1}, \ldots, z_{m}, \alpha_{1}, \ldots, \alpha_{m}\right) \in W$ for all $z_{1}, \ldots, z_{m} \in W_{1}$ and $\left(\alpha_{1}, \ldots, \alpha_{m}\right) \in S_{m}$. The continuity of $f$ in the first variable at $\left(x_{0}, y_{0}\right)$ implies the existence of a neighborhood $U$ of $x_{0}$ in $X$ such that $f\left(x, y_{0}\right) \in W_{1}$ for every $x \in U$. According to (4.1) there is a number $n_{0}$ such that $x_{i, n} \in U$ for all $n \geq n_{0}$ and $i \in I_{n}$ with $x_{0} \in \operatorname{supp} \varphi_{i, n}$.

We will show that $f_{n}\left(x_{0}, y_{0}\right) \in W$ for all $n \geq n_{0}$. Take $n \geq n_{0}$ and set

$$
I=\left\{i \in I_{n}: x_{0} \in \operatorname{supp} \varphi_{i, n}\right\} .
$$

Let $I=\left\{i_{1}, i_{2}, \ldots i_{k}\right\}$, where $i_{1}<i_{2}<\cdots<i_{k}$ and $k \leq m$. Denote $\alpha_{1}=\varphi_{i_{1}, n}\left(x_{0}\right), \ldots, \alpha_{k}=\varphi_{i_{k}, n}\left(x_{0}\right)$, $z_{1}=f\left(x_{i_{1}, n}, y_{0}\right), \ldots, z_{k}=f\left(x_{i_{k}, n}, y_{0}\right)$. Then $z_{1}, \ldots z_{k} \in W_{1}$. Taking into account Proposition $2.3(i)$, we have

$$
\begin{gathered}
f_{n}\left(x_{0}, y_{0}\right)=\lambda_{k}\left(z_{1}, \ldots, z_{k}, \alpha_{1}, \ldots \alpha_{k}\right)= \\
=\lambda_{m}\left(z_{1}, \ldots, z_{k}, z_{0}, \ldots, z_{0}, \alpha_{1}, \ldots \alpha_{k}, 0, \ldots, 0\right) \in W .
\end{gathered}
$$


A topological space $X$ is strongly countably dimensional if there exists a sequence $\left(X_{n}\right)_{n=1}^{\infty}$ of sets $X_{n} \subseteq X$ such that $X=\bigcup_{n=1}^{\infty} X_{n}$ and $\operatorname{dim} X_{n}<n$ for every $n \in \mathbb{N}$, where by $\operatorname{dim} Y$ we denote Cech-Lebesgue $\operatorname{dimension}$ of $Y$ (see [3, p. 564]).

Theorem 5.3. Let $X$ be a Hausdorff paracompact strongly countably dimensional strong PP-space, $Y$ a topological space, $(Z, \lambda)$ an equiconnected space and $0 \leq \alpha<\omega_{1}$. Then $(X, Y, Z)$ is a Rudin $\alpha$-triple.

Proof. Let $f \in C \bar{B}_{\alpha}(X \times Y, Z)$. Using Definition 4.2 we choose a sequence $\left.\left(\varphi_{i, n}: i \in I_{n}\right)\right)_{n=1}^{\infty}$ of locally finite partitions of unity $\left(\varphi_{i, n}: i \in I_{n}\right)$ on $X$ and a sequence $\left(\left(x_{i, n}: i \in I_{n}\right)\right)_{n=1}^{\infty}$ of families of points $x_{i, n} \in X_{B_{\alpha}}(f)$ such that (4.1) holds. By [4, Theorem 5.1.10] for every $n \in \mathbb{N}$ there exists a locally finite cover $\left(U_{j, n}: j \in J_{n}\right)$ of $X$ which refines $\left(\operatorname{supp} \varphi_{i, n}: i \in I_{n}\right)$ and such that for every $m \in \mathbb{N}$ and $x \in X_{m}$ there is a neighborhood $U$ of $x$ with $\left|\left\{j \in J_{n}: U \cap U_{j, n} \neq \emptyset\right\}\right| \leq m$. For every $n \in \mathbb{N}$ choose a locally finite partition of unity $\left(\psi_{j, n}: j \in J_{n}\right)$ on $X$ subordinated to $\left(U_{j, n}: j \in J_{n}\right)$. For every $j \in J_{n}$ denote by $u_{j, n}$ such an element $x_{i, n}$ that $U_{j, n} \subseteq \operatorname{supp} \varphi_{i, n}$. Let us observe that the sequences $\left(\left(\psi_{j, n}: j \in J_{n}\right)\right)_{n=1}^{\infty}$ and $\left(\left(u_{j, n}: j \in J_{n}\right)\right)_{n=1}^{\infty}$ satisfy the conditions of Theorem 5.2, hence $f \in B_{\alpha+1}(X \times Y, Z)$.

The following corollary generalizes Theorem 1.3 .

Corollary 5.4. Let $X$ be a strongly countably dimensional metrizable space, $Y$ a topological space, ( $Z, \lambda)$ an equiconnected space. Then $(X, Y, Z)$ is a Rudin $\alpha$-triple for every $0 \leq \alpha<\omega_{1}$.

\section{THE CASE $\alpha>0$}

Definition 6.1. A topological space $X$ is contractible if there exist a point $x^{*} \in X$ and a continuous function $\gamma: X \times[0,1] \rightarrow X$ such that $\gamma(x, 0)=x$ and $\gamma(x, 1)=x^{*}$ for every $x \in X$. A contractible space $X$ with such a function $\gamma$ and such a point $x^{*}$ we denote by $\left(X, \gamma, x^{*}\right)$.

Clearly, every equiconnected space $(X, \lambda)$ is contractible.

Proposition 6.2. Let $X$ and $Y$ be topological spaces, $\left(Z, \gamma, z^{*}\right)$ a contractible space, $0 \leq \alpha<\omega_{1}$, $\left(g_{i}\right)_{i \in I} a$ family of mappings $g_{i} \in B_{\alpha}(Y, Z)$ and let $\left(\varphi_{i}\right)_{i \in I}$ be a family of continuous functions $\varphi_{i}: X \rightarrow[0,1]$ such that the family $\left(\operatorname{supp} \varphi_{i}\right)_{i \in I}$ is discrete in $X$. Then the mapping $f: X \times Y \rightarrow Z$,

$$
f(x, y)= \begin{cases}\gamma\left(g_{i}(y), 1-\varphi_{i}(x)\right), & \text { if } x \in \operatorname{supp} \varphi_{i} \text { for some } i \in I, \\ z^{*}, & \text { else, }\end{cases}
$$

is of the Baire class $\alpha$.

Proof. We first consider the case when $\alpha=0$. Fix a point $x_{0} \in X$. Since the family $\left(\operatorname{supp} \varphi_{i}\right)_{i \in I}$ is discrete, we can choose a neighborhood $U_{0}$ of $x_{0}$ such that $\left\{i \in I: U_{0} \cap \operatorname{supp} \varphi_{i} \neq \emptyset\right\} \subseteq\{j\}$ for some $j \in I$. Then $f(x, y)=\gamma\left(g_{j}(y), 1-\varphi_{j}(x)\right)$ for all $x \in U_{0}$ and $y \in Y$. Hence, $f$ is continuous at every point of $\left\{x_{0}\right\} \times Y$.

Further, we use the induction on $\alpha$ analogously as in the proof of Theorem 3.2 .

Recall that a subset $A$ of a topological space $X$ is a zero (co-zero) set if there exists a continuous function $f: X \rightarrow[0,1]$ such that $A=f^{-1}(0)\left(A=f^{-1}((0,1])\right)$. A set $A$ is functionally ambiguous if there are two sequences $\left(A_{n}\right)_{n=1}^{\infty}$ and $\left(B_{n}\right)_{n=1}^{\infty}$ of zero-sets $A_{n}$ and co-zero sets $B_{n}$ such that $A=\bigcup_{n=1}^{\infty} A_{n}=\bigcap_{n=1}^{\infty} B_{n}$.

Lemma 6.3. Let $X$ be a topological space and $\mathcal{G}$ be a locally finite cover of $X$ by co-zero sets. Then there exists a disjoint locally finite cover of $X$ by functionally ambiguous sets which refines $\mathcal{G}$.

Proof. Write $\mathcal{G}=\left\{G_{\alpha}: 0 \leq \alpha<\beta\right\}$, where $\beta$ is an ordinal. Set $A_{0}=G_{0}$. For every $0<\alpha<\beta$ let $A_{\alpha}=G_{\alpha} \backslash \bigcup_{\xi<\alpha} G_{\xi}$. Since $\mathcal{G}$ is locally finite, the set $\bigcup_{\xi<\alpha} G_{\xi}$ is a co-zero set. Then $A_{\alpha}$ is functionally ambiguous set as the difference of co-zero sets. Clearly, the family $\mathcal{A}=\left(A_{\alpha}: 0 \leq \alpha<\beta\right)$ is to be found.

Definition 6.4. A topological space $X$ is said to be weakly collectionwise normal if for an arbitrary discrete family $\left(F_{s}: s \in S\right)$ of zero sets in $X$ there exists a discrete family $\left(U_{s}: s \in S\right)$ of co-zero sets in $X$ such that $F_{s} \subseteq U_{s}$ for all $s \in S$.

Let us observe that every collectionwise normal space is weakly collectionwise normal. Remark also that every metrizable space or, more general, a space equipped with the topology generated by a pseudo-metric, is weakly collectionwise normal. 
Proposition 6.5. Let $0<\alpha<\omega_{1}$, let $X$ be a weakly collectionwise normal space, $Y$ a topological space, $Z$ a contractible space, $\left(X_{s}: s \in S\right)$ a disjoint locally finite cover of $X$ by functionally ambiguous sets, $g_{s} \in B_{\alpha}(Y, Z)$ for every $s \in S$ and $f(x, y)=g_{s}(y)$ if $(x, y) \in X_{s} \times Y$. Then $f \in B_{\alpha}(X \times Y, Z)$.

Proof. For every $s \in S$ there exists an increasing sequence $\left(F_{s, n}\right)_{n=1}^{\infty}$ of zero sets such that $X_{s}=\bigcup_{n=1}^{\infty} F_{s, n}$. Since for every $n \in \mathbb{N}$ all the sets $F_{s, n}$ are closed, every family $\left(F_{s, n}: s \in S\right)$ is discrete in $X$. Taking into account that $X$ is weakly collectionwise normal, for every $n \in \mathbb{N}$ we choose a discrete family $\left(U_{s, n}: s \in S\right)$ of co-zero sets in $X$ such that $F_{s, n} \subseteq U_{s, n}$ for all $s \in S$. Let $\varphi_{s, n} \in C(X,[0,1])$ be such functions that $F_{s, n}=\varphi_{s, n}^{-1}(1)$ and $U_{s, n}=\varphi_{s, n}^{-1}((0,1])$ for all $(s, n) \in S \times \mathbb{N}$.

We choose an increasing sequence $\left(\alpha_{n}\right)_{n=1}^{\infty}$ of ordinals $\alpha_{n}<\alpha$ such that $\alpha=\lim _{n \rightarrow \infty}\left(\alpha_{n}+1\right)$ and for every $s \in S$ choose a sequence of functions $g_{s, n} \in B_{\alpha_{n}}(Y, Z)$ which is pointwise convergent to $g_{s}$ on $Y$.

Let $\gamma \in C(Z \times[0,1], Z)$ and $z_{0} \in Z$ be such that $\gamma(z, 0)=z$ and $\gamma(z, 1)=z_{0}$ for all $z \in Z$. Define a function $f_{n}: X \times Y \rightarrow Z$ in the following way:

$$
f_{n}(x, y)= \begin{cases}\gamma\left(g_{s, n}(y), 1-\varphi_{s, n}(x)\right), & \text { if } x \in U_{s, n} \text { for some } s \in S, \\ z_{0}, & \text { else. }\end{cases}
$$

According to Proposition 6.2, $f_{n} \in B_{\alpha_{n}}(X \times Y, Z)$ for every $n \in \mathbb{N}$.

It remains to prove that $f_{n} \rightarrow f$ on $X \times Y$. Indeed, let $(x, y) \in X \times Y$. Then there exists $s \in S$ such that $x \in X_{s}$. Since the sequence $\left(F_{s, n}\right)_{n=1}^{\infty}$ increases, there exists a number $n_{0}$ such that $x \in F_{s, n}$ for all $n \geq n_{0}$. Then $f_{n}(x, y)=\gamma\left(g_{s, n}(y), 0\right)=g_{s, n}(y)$ for all $n \geq n_{0}$. Hence,

$$
f_{n}(x, y) \rightarrow g_{s}(y)=f(x, y) .
$$

Therefore, $f \in B_{\alpha}(X \times Y, Z)$.

Theorem 6.6. Let $X$ be a strong PP-space, $Y$ a topological space, $Z$ a contractible space and $0<\alpha<\omega_{1}$. Then $(X, Y, Z)$ is a Rudin $\alpha$-triple.

Proof. Let $f \in C \bar{B}_{\alpha}(X \times Y, Z)$. For the set $X_{B_{\alpha}}(f)$ there exist a sequence $\left(\left(\varphi_{i, n}: i \in I_{n}\right)\right)_{n=1}^{\infty}$ of locally finite partitions of unity on $X$ and a sequence $\left(\left(x_{i, n}: i \in I_{n}\right)\right)_{n=1}^{\infty}$ of families of points of $X_{B_{\alpha}}(f)$ such that condition (4.1) holds.

In accordance with [10, Proposition 3.2] there exists a pseudo-metric on $X$ such that all the functions $\varphi_{i, n}$ are continuous with respect to this pseudo-metric. Denote by $\mathcal{T}$ the topology on $X$ generated by the pseudo-metric. Obviously, the topology $\mathcal{T}$ is weaker than the initial one and all the sets $\operatorname{supp} \varphi_{i, n}$ are $\mathcal{T}$-open. Using the paracompactness of $(X, \mathcal{T})$, for every $n$ we choose a locally finite open cover $\mathcal{V}_{n}$ which refines the open cover $\left(\operatorname{supp} \varphi_{i, n}: i \in I_{n}\right)$. By Lemma 6.3 for every $n$ there exists a disjoint locally finite cover $\mathcal{A}_{n}=\left(A_{s, n}: s \in S_{n}\right)$ of $(X, \mathcal{T})$ by ambiguous sets which refines $\mathcal{V}_{n}$. For every $s \in S_{n}$ we choose any $i(s) \in I_{n}$ such that $A_{s, n} \subseteq$ $\operatorname{supp} \varphi_{i(s), n}$. For all $n$ and $(x, y) \in X \times Y$ let

$$
f_{n}(x, y)=f^{x_{i(s), n}}(y) \text {, if } x \in A_{s, n} .
$$

Since $f^{x_{i(s), n}} \in B_{\alpha}(Y, Z)$ for every $s \in S_{n}$ and $(X, \mathcal{T})$ is weakly collectionwise normal, Proposition 6.5 implies that $f_{n} \in B_{\alpha}((X, \mathcal{T}) \times Y, Z) \subseteq B_{\alpha}(X \times Y, Z)$ for every $n \in \mathbb{N}$.

Now we show that $f_{n} \rightarrow f$ on $X \times Y$. Fix $(x, y) \in X \times Y$. There exists a sequence $\left(s_{n}\right)_{n=1}^{\infty}, s_{n} \in S_{n}$, such that $x \in A_{s_{n}} \subseteq \operatorname{supp} \varphi_{i\left(s_{n}\right), n}$. Condition (4.1) implies that $x_{i\left(s_{n}\right), n} \rightarrow x$. Since $f$ is continuous in the first variable,

Hence, $f \in B_{\alpha+1}(X \times Y, Z)$.

$$
f_{n}(x, y)=f\left(x_{i\left(s_{n}\right), n}, y\right) \rightarrow f(x, y) .
$$

\section{Vertically nearly separately continuous mappings Which do not belong to the first Baire} CLASS

Let $X=\{0\} \cup \bigcup_{n=1}^{\infty} X_{n}$, where $X_{n}=\left\{\frac{1}{n}\right\} \cup \bigcup_{m=n^{2}}^{\infty}\left\{\frac{1}{n}+\frac{1}{m}\right\}$. We define a topology on $X$ in the following way. All points of the form $\frac{1}{n}+\frac{1}{m}$ will be isolated points of $X$. The base of neighborhoods of a point $\frac{1}{n}$ are the sets of the form $X_{n} \backslash \bigcup_{m=n^{2}}^{k}\left\{\frac{1}{n}+\frac{1}{m}\right\}, k=n^{2}, n^{2}+1, \ldots$. Finally, as neighborhoods of 0 we take all the sets obtained from $X$ by removing a finite number of $X_{n}$ 's and a finite number of points of the form $\left\{\frac{1}{n}+\frac{1}{m}\right\}$ in all the remaining $X_{n}$ 's. The space $X$ with this topology is a sequential space that is not a Fréchet space [3, Example 1.6.19]. 
The following example is a development of Example 5.7 from [1] and shows that the condition that $X$ is strong PP-space is essential in Theorems 4.5,5.3 and 6.6. It then follows that Theorem 1.6 cannot be generalized to Rudin triples.

Example 7.1. Let $0 \leq \alpha<\omega_{1}$, let $Y$ and $Z$ be topological spaces such that $B_{\alpha+2}(Y, Z) \backslash B_{\alpha+1}(Y, Z) \neq \emptyset$. Then there exists a function $f \in C \bar{B}_{\alpha}(X \times Y, Z)$, which is not a pointwise limit of a sequence of functions $f_{n} \in C B_{\alpha}(X \times Y, Z)$.

Proof. Let $g \in B_{\alpha+2}(Y, Z) \backslash B_{\alpha+1}(Y, Z)$. Then there exist a sequence $\left(g_{n}\right)_{n=1}^{\infty}$ of mappings $g_{n} \in B_{\alpha+1}(Y, Z)$ and a family $\left(g_{n m}: n, m \in \mathbb{N}\right)$ of mappings $g_{n m} \in B_{\alpha}(Y, Z)$ such that $g_{n}(y) \rightarrow g(y)$ for all $y \in Y$ and $g_{n m}(y) \rightarrow g_{n}(y)$, when $m \rightarrow \infty$, for all $n \in \mathbb{N}$ and $y \in Y$. Let $K=\left\{(n, m): n \in \mathbb{N}, m \in \mathbb{N}, m \geq n^{2}\right\}, x_{0}=0$, $x_{n}=\frac{1}{n}, x_{n m}=\frac{1}{n}+\frac{1}{m}$, where $(n, m) \in K$, and consider the function $f: X \times Y \rightarrow Z$,

$$
f(x, y)= \begin{cases}g(y), & x=x_{0}, \\ g_{n}(y), & x=x_{n}, \\ g_{n m}(y), & x=x_{n m} .\end{cases}
$$

It is easy to verify that $X_{B_{\alpha}}(f)=\left\{x_{n m}:(n, m) \in K\right\}, \overline{X_{B_{\alpha}}(f)}=X$ and $f_{y} \in C(X, Z)$ for every $y \in Y$. Hence, $f \in C \bar{B}_{\alpha}(X \times Y, Z)$.

Assume that there exists a sequence of functions $f_{n} \in C B_{\alpha}(X \times Y, Z)$ which pointwise converges to $f$. Then $f_{n}(0, y) \rightarrow f(0, y)=g(y)$ for all $y \in Y$, i.e. $g \in B_{\alpha+1}(Y, Z)$, a contradiction.

Lemma 7.2. Let $X$ be a topological space, $a \in \mathbb{R}$, let $F, H$ be zero sets in $X, G$ a co-zero set in $X$ such that $H \subseteq F \cap G$. Then there exists a function $f: X \times \mathbb{R} \rightarrow \mathbb{R}$ with the followings properties:

(1) $f$ is continuous in the first variable;

(2) $f$ is jointly continuous on $(x, y) \in(X \backslash F) \times \mathbb{R}$;

(3) $f(x, y)=0$ if $(x, y) \in(X \backslash G) \times \mathbb{R}$ or $(x, y) \in F \times(\mathbb{R} \backslash\{a\})$;

(4) $f(x, a)=1$ if $x \in H$.

Proof. We pick continuous functions $\varphi: X \rightarrow[0,1]$ and $\psi: X \rightarrow[0,1]$ such that $H=\varphi^{-1}(1), X \backslash G=\varphi^{-1}(0)$ and $F=\psi^{-1}(0)$. Moreover, consider the continuous function $g: \mathbb{R} \times(0,+\infty) \rightarrow[0,1]$,

$$
g(u, v)= \begin{cases}\cos \frac{\pi(u-a)}{2 v}, & |u-a| \leq v, \\ 0, & |u-a|>v .\end{cases}
$$

Now consider the continuous function $f: X \times \mathbb{R} \rightarrow \mathbb{R}$,

$$
f(x, y)= \begin{cases}\varphi(x) g(y, \psi(x)), & x \in X \backslash F, \\ \varphi(x) \chi_{\{a\}}(y), & x \in F,\end{cases}
$$

where by $\chi_{\{a\}}$ we denote the characteristic function of the set $\{a\}$. Let us observe that (1) and (2) follow from the continuity of functions $\varphi, \psi$ and $g$ and the equality $\lim _{v \rightarrow 0} g(u, v)=\chi_{\{a\}}(u)$ for every $u \in \mathbb{R}$. Conditions $(3)$ and (4) immediately follow from the definition of $f$.

Denote by $\mathbb{R}^{\infty}$ the collection of all sequences with finite support, i.e. sequences of the form $\left(\xi_{1}, \xi_{2}, \ldots, \xi_{n}, 0,0, \ldots\right)$, where $\xi_{1}, \xi_{2}, \ldots, \xi_{n} \in \mathbb{R}$. Clearly, $\mathbb{R}^{\infty}$ is a linear subspace of the space $\mathbb{R}^{\mathbb{N}}$ of all sequences. Denote by $E$ the set of all sequences $e=\left(\varepsilon_{n}\right)_{n=1}^{\infty}$ of positive reals $\varepsilon_{n}$ and let

$$
U_{e}=\left\{x=\left(\xi_{n}\right)_{n=1}^{\infty} \in \mathbb{R}^{\infty}:(\forall n \in \mathbb{N})\left(\left|\xi_{n}\right| \leq \varepsilon_{n}\right)\right\}
$$

We consider on $\mathbb{R}^{\infty}$ the topology in which the system $\mathcal{U}_{0}=\left\{U_{e}: e \in E\right\}$ forms the base of neighborhoods of zero.

The following example shows that the analog of Theorem 5.3 is not true for strongly countably dimensional locally convex Hausdorff paracompact spaces.

Example 7.3. Let $X=\mathbb{R}^{\infty}$ and $Y=Z=\mathbb{R}$. Then there exists a function $f \in C \bar{C}(X \times Y, Z)$ which is not a pointwise limit of a sequence of separately continuous functions.

Proof. For every $n \in \mathbb{N}$ let

$$
\begin{gathered}
F_{n}=\left\{\left(\xi_{1}, \ldots, \xi_{n}, 0,0 \ldots\right) \in X: \max \left\{\left|\xi_{1}\right|, \ldots,\left|\xi_{n}\right|\right\} \leq \frac{1}{n}\right\} \\
\tilde{F}_{n}=\left\{\left(\xi_{1}, \ldots, \xi_{n}, \xi_{n+1}, \ldots\right) \in X: \max \left\{\left|\xi_{1}\right|, \ldots,\left|\xi_{n}\right|\right\} \leq \frac{1}{n}\right\} \\
G_{n}=\left\{\left(\xi_{1}, \ldots, \xi_{n}, \xi_{n+1}, \ldots\right) \in X: \max \left\{\left|\xi_{1}\right|, \ldots,\left|\xi_{n}\right|\right\}<\frac{1}{n-\frac{1}{2}}\right\}
\end{gathered}
$$


and

$$
H_{n}=\bigcap_{m=n}^{\infty}\left(\bigcup_{k=n}^{m} F_{k} \cup \tilde{F}_{m}\right) .
$$

It is easily seen that all the sets $H_{n}$ are zero sets, and all the sets $G_{n}$ are co-zero sets.

Let $\mathbb{Q}=\left\{r_{n}: n \in \mathbb{N}\right\}$, where $r_{n} \neq r_{m}$ for $n \neq m$. Fix $n \in \mathbb{N}$. For the space $X$, a number $a=r_{n}$ and for sets $F=H_{1}, H_{n}$ and $G_{n}$ there exists a function $f_{n}: X \times \mathbb{R} \rightarrow \mathbb{R}$ which satisfies (1) - (4) of Lemma 7.2. We set $f(x, y)=\sum_{n=1}^{\infty} f_{n}(x, y)$. We will show that $f \in C \bar{C}(X \times Y, Z)$, but $f$ is not a pointwise limit of a sequence of separately continuous functions.

Remark that $f_{n}(x, y)=\chi_{r_{n}}(y)$ for all $n \in \mathbb{N}, y \in Y$ and $x \in H_{n}$. Then for the point $x_{0}=(0,0, \ldots) \in \bigcap_{n=1}^{\infty} H_{n}$ the function $f^{x_{0}}$ is the Dirichlet function, which is not of the first Baire class. Hence, $f$ is not a pointwise limit of a sequence of separately continuous functions.

Now we prove that $X \backslash F \subseteq X_{C}(f)$. Let $x_{1} \in X \backslash F$. Since $x_{1} \neq x_{0}$, there exist a neighborhood $U \subseteq X \backslash F$ of $x_{1}$ and a number $n_{0} \in \mathbb{N}$ such that $U \cap G_{n}=\emptyset$ for all $n \geq n_{0}$. Then, taking into account condition (3), for every $x \in U$ we have $f(x, y)=\sum_{k=1}^{n_{0}} f_{k}(x, y)$ for all $y \in Y$. By condition $(2), f$ is continuous on $U \times Y$, in particular, $x_{1} \in X_{C}(f)$. Since $\overline{X \backslash F}=X, \overline{X_{C}(f)}=X$.

It remains to show that $f$ is continuous in the first variable. Fix $y_{0} \in Y$. Since the sequence $\left(G_{n}\right)_{n=1}^{\infty}$ is decreasing and $\left\{x_{0}\right\}=\bigcap_{n=1}^{\infty} \overline{G_{n}}$, conditions (3) and (1) imply that the function $f_{y_{0}}$ is continuous at all points $x \neq x_{0}$. Notice that a given function $g: \mathbb{R}^{\infty} \rightarrow \mathbb{R}$ is continuous if and only if the restriction of $g$ to every space $X_{n}=\left\{\left(\xi_{1}, \ldots, \xi_{n}, 0 \ldots\right): \xi_{1}, \ldots, \xi_{n} \in \mathbb{R}\right\}$ is continuous. Therefore, it is sufficient to show that every restriction of $f_{y_{0}}$ to $X_{n}$ is continuous at $x_{0}$. Choose a number $m \in \mathbb{N}$ such that $y_{0} \notin\left\{r_{k}: k \geq m\right\}$. Then by condition (3), $f\left(x, y_{0}\right)=\sum_{k=1}^{m} f_{k}\left(x, y_{0}\right)$ for all $x \in F$. Since for every $n \in \mathbb{N}$ the set $F_{n}=F \cap X_{n}$ is a neighborhood of $x_{0}$ in $X_{n}$, one has that (1) imply the continuity of the restriction of $f_{y_{0}}$ on $X_{n}$ at the point $x_{0}$.

Remark that the spaces from Example 7.1 and Example 7.3 are PP-spaces. But combining these examples and Theorem 4.5 implies that they are not strong PP-spaces.

Let us observe that the inclusion $C \bar{C}(X \times Y, \mathbb{R}) \subseteq B_{1}(X \times Y, \mathbb{R})$ for any topological space $Y$ does not imply that $X$ is a strong PP-space. For example, the inclusion holds for any topological space $X$ on which every continuous real-valued function is constant. These spaces, in general, need not by regular (see [3, p. 119]) and (strong) $P P$-spaces. Therefore, it is natural to ask the following question.

Question 7.4. Does there exist a completely regular space $X$ which is not a (strong) PP-space and $C \bar{C}(X \times Y, \mathbb{R}) \subseteq B_{1}(X \times Y, \mathbb{R})$ for any topological space $Y$ ?

\section{REFERENCES}

[1] T. Banakh, (Metrically) quarter-stratifiable spaces and their applications, Math. Studii 18(1) (2002), 10-28.

[2] M. Burke, Borel measurability of separately continuous functions, Topology Appl. 129 (1) (2003), 29-65.

[3] R. Engelking, General Topology, Revised and completed edition, Heldermann Verlag, Berlin (1989).

[4] R. Engelking, Theory of dimensions, finite and infinite, Revised and completed edition. Heldermann Verlag, Lemgo (1995).

[5] H. Hahn, Reelle Funktionen.1.Teil. Punktfunktionen, Leipzig: Academische Verlagsgesellscheft M.B.H. (1932).

[6] A. Kalancha, V. Maslyuchenko, Čech-Lebesgue dimension and Baire classification of vector-valued separately continuous mappings, Ukr.Math.J. 55 (11) (2003), 1596-1599. (in Ukrainian)

[7] O. Karlova, Baire classification of mappings which are continuous in the first variable and of the functional class $\alpha$ in the second one, Math. Bull. NTSH. 2 (2005), 98-114.

[8] H. Lebesgue, Sur l'approximation des fonctions, Bull. Sci. Math. 22 (1898), 278-287.

[9] W. Moran, Separate continuity and supports of measures, J. London Math. Soc. 44 (1969), 320-324.

[10] V. Mykhaylyuk, Baire classification of separately continuous functions and Namioka property, Ukr. Math. Bull. 5 (2) (2008), 203-218.

[11] W. Rudin, Lebesgue first theorem, Math. Analysis and Applications, Part B. Edited by Nachbin. Adv. in Math. Supplem. Studies 78, Academic Press (1981), 741-747.

[12] O. Sobchuk, Baire classification and Lebesgue spaces, Bull. of Chernivtsi Nat. Univ. Mathematics, 111 (2001), 110-112

[13] O. Sobchuk, PP-spaces and Baire classification, International Conference on Functional Analysis and its Applications, dedicated to the 110th anniversary of Stefan Banach. Book of abstracts (2002), 189.

[14] G. Vera, Baire mesurability of separately continuous functions, Quart. J. Math. Oxford 39 (2) (1988), 109-116. 